\title{
Association of a Polyadenylation Polymorphism in the Serotonin Transporter and Panic Disorder
}

\author{
Sandeep Gyawali, Ryan Subaran, Myrna M. Weissman, Dylan Hershkowitz, Morgan C. McKenna, \\ Ardesheer Talati, Abby J. Fyer, Priya Wickramaratne, Phillip B. Adams, Susan E. Hodge, Carl J. Schmidt, \\ Michael J. Bannon, and Charles E. Glatt
}

Background: Genetic markers in the serotonin transporter are associated with panic disorder (PD). The associated polymorphisms do not include the serotonin transporter-linked polymorphic region and display no obvious functional attributes. A common polymorphism (rs3813034) occurs in one of the two reported polyadenylation signals for the serotonin transporter and is in linkage disequilibrium with the PD-associated markers. If functional, rs3813034 might be the risk factor that explains the association of the serotonin transporter and PD.

Methods: Quantitative polymerase chain reaction on human brain samples $(n=65)$ and lymphoblast cultures $(n=71)$ was used to test rs3813034 for effects on expression of the polyadenylation forms of the serotonin transporter. rs3813034 was also tested for association in a sample of PD cases $(n=307)$ and a control sample $(n=542)$ that has similar population structure.

Results: The balance of the two polyadenylation forms of the serotonin transporter is associated with rs3813034 in brain ( $p<.001)$ and lymphoblasts $(p<.001)$. The balance of the polyadenylation forms is also associated with gender in brain only $(p<.05)$. Association testing of rs3813034 in PD identified a significant association $(p=.0068)$ with a relative risk of 1.56 and 1.81 for the heterozygous and homozygous variant genotypes, respectively.

Conclusions: rs3813034 is a functional polymorphism in the serotonin transporter that alters the balance of the two polyadenylation forms of the serotonin transporter. rs3813034 is a putative risk factor for PD and other behavioral disorders that involve dysregulation of serotonergic neurotransmission.

Key Words: Gender, genetic, panic disorder, polyadenylation, polymorphism, serotonin transporter

$\mathrm{P}$ anic disorder (PD) is a well-characterized anxiety disorder that displays a substantial genetic component and a gender difference of roughly twofold increased prevalence in women (1-6). Alterations in serotonergic neurotransmission and the serotonin transporter (SERT) in particular have been implicated in the pathophysiology of PD through several lines of evidence (7). First, the selective serotonin reuptake inhibitors (SSRIs) that target SERT are among the most commonly used and most effective treatments for PD (8-10). Second, genetically modified mice that underexpress SERT display an anxiety-like phenotype, and these animals are unresponsive to SSRIs (11-13). Finally, in vivo human studies of PD subjects have found decreased SERT expression in brain and platelets and increased serotonin turnover that is normalized by SSRIs $(14,15)$.

A number of genetic association studies of PD have focused on a polymorphism in a putative regulatory region of the human gene for SERT (SLC6A4) known as the serotonin transporterlinked polymorphic region (5HTTLPR) (16-18). The 5HTTLPR has been associated with trait anxiety, amygdala activation in

From the Department of Psychiatry (SG, DH, MCM, CEG), Weill Medical College of Cornell University; Department of Psychiatry (RS, MMW, AT, AJF, PW, SEH), College of Physicians and Surgeons; Mailman School of Public Health (MMW, PW); Division of Statistical Genetics (PBA, SEH), Department of Biostatistics, Columbia University, New York, New York; Division of Epidemiology (MMW, AT, PBA, SEH); Division of Clinical Therapeutics (AJF), New York State Psychiatric Institute, New York, New York; and the Departments of Pathology (CJS) and Pharmacology (MJB), and the Center for Molecular Medicine and Genetics, Wayne State University School of Medicine, Detroit, Michigan.

Address correspondence to Charles E. Glatt, M.D., Ph.D., Room LC929A, 1300 York Avenue, New York, NY 10065; E-mail: ceg2004@med.cornell.edu. Received Jul 27, 2009; revised Sep 17, 2009; accepted Oct 11, 2009. response to fearful stimuli, and major depression in the setting of stressful life events $(16,19,20)$. Most studies of the 5HTTLPR in PD have not identified significant associations (21-30), and a formal meta-analysis of these studies found no evidence for association between the 5HTTLPR and PD (31). More recently a comprehensive screen of markers spanning the SERT locus identified several single nucleotide polymorphisms (SNPs) that are associated with PD, suggesting that a functional polymorphism elsewhere in the gene might contribute to risk for PD (32). These associated polymorphisms are all in linkage disequilibrium (LD) with each other in a haplotype block at the $3^{\prime}$ end of SLC6A4 in Caucasian populations but are not in strong LD with the 5HTTLPR (Figure S1 in Supplement 1). None of the PDassociated polymorphisms display obvious functional properties on the basis of their location in SLC6A4.

The $3^{\prime}$ untranslated region (UTR) of the SERT messenger RNA (mRNA) exists in two reported forms that differ by the presence or absence of a 123-base pair (bp) element (Figure 1) (33,34). The more $3^{\prime}$ (distal) of the polyadenylation signals for these two forms contains a common SNP (rs3813034) (33,34). rs3813034 was not tested directly in the previous report, but it is in the haplotype block that is associated with PD (S1), and because of its location in the six nucleotide distal polyadenylation signal for the SERT mRNA might have functional effects on the usage of that signal. If so, rs3813034 might be the risk factor that explains the association of SLCGA4 to PD. To test this hypothesis we characterized the effects of rs3813034 on the polyadenylation of SERT in physiological tissue and heterologous expression experiments to determine whether it is functional. We also tested rs3813034 for association to PD in the sample that has previously displayed association to SLCGA4 to which additional cases and control subjects have been added. Our molecular characterization of rs3813034 allowed us to make an a priori prediction of which allele should be associated with increased PD risk, which we tested in the association analysis (35). 


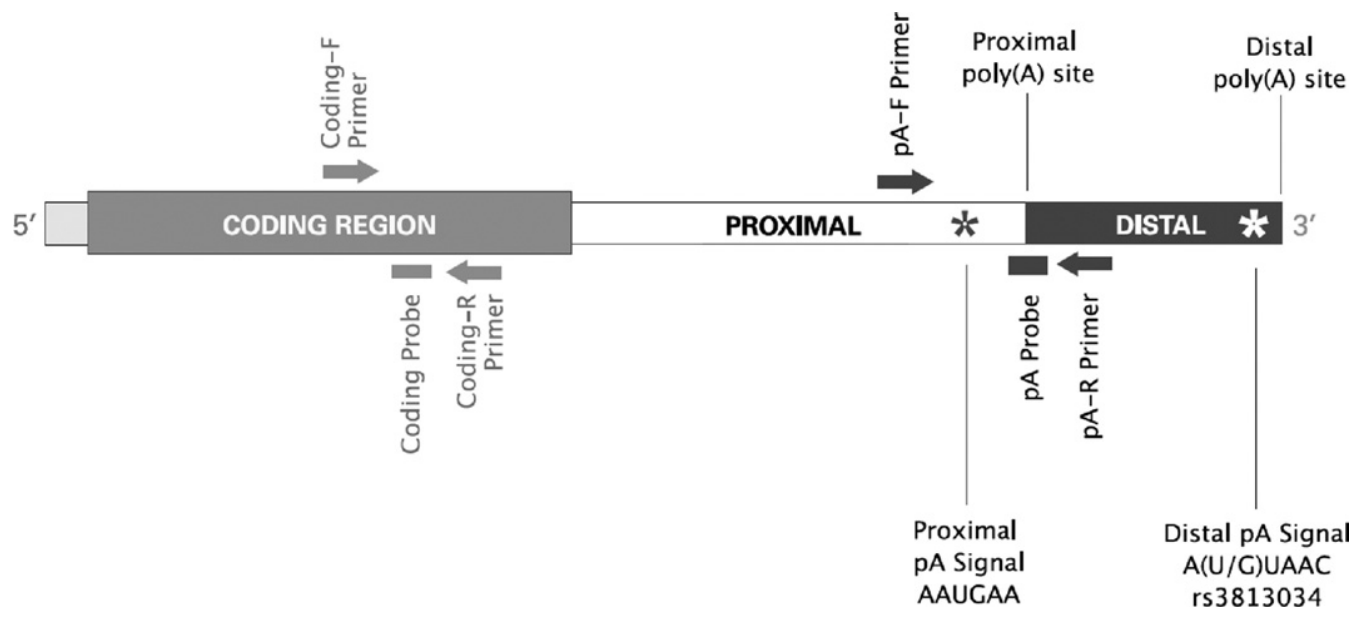

Figure 1. Schematic representation of the serotonin transporter message (mRNA) and quantitative polymerase chain reaction (qPCR) assays. The $5^{\prime}$ and $3^{\prime}$ untranslated regions are presented as narrow boxes. The protein-coding region is presented as a wide box. Primers and probes for the $\mathrm{qPCR}$ assays are placed in the location of their hybridization positions. Probes are represented on the strand to which they hybridize.

\section{Methods and Materials}

\section{Subjects}

Molecular studies of SERT polyadenylation used population samples of postmortem human brain and Epstein-Barr virustransformed lymphoblast cultures.

Sixty-five postmortem human midbrain specimens (42 men and 23 women; 33 African American, 31 European American, 1 Hispanic American) were obtained during routine autopsy as described previously $(36,37)$. Brains were dissected to obtain coronal brain sections taken between the level of the red nucleus/substantia nigra and midpons, which included the raphe nuclei. Postmortem interval for brains ranged from 4 hours to 20 hours, and sample pHs were all $>6.1$ to ensure minimal perimortem RNA degradation (38). Tissue was rapidly dissected and frozen. Tissue blocks were stored at $-80^{\circ} \mathrm{C}$. Routine toxicology for drugs of abuse were performed as part of all autopsies.

Seventy-one lymphoblast cultures from European American Centre d'Etude du Polymorphisme Humain (CEPH) subjects ( 42 men and 29 women) were obtained from the Coriell Cell Repository. Lymphoblast cultures were grown under recommended conditions (Methods and Materials in Supplement 1).

The PD association sample consisted of 307 PD cases and 544 ethnically matched control subjects. This sample includes all the Caucasian subjects from the previous association study and additional cases and control subjects that have been recruited since that report and were added to increase power $(32,35)$. The PD cases were recruited from across the United States through community-based mailings, media articles, or websites describing the study and were ascertained with the Schedule for Affective Disorders and Schizophrenia Lifetime Version modified for the study of anxiety disorders and updated for the DSM IV (SADS-LA IV) $(39,40)$. Family history of psychopathology was determined with the Family History Screen with the proband as the informant (41). The PD cases met criteria for probable or definite PD (42). Subjects with a history of schizophrenia, bipolar disorder, and antisocial personality disorder were excluded, as were subjects whose panic symptoms might be explained by a medical or neurological disorder. Of 794 subjects interviewed, 392 were classified as PD or PD plus social anxiety disorder. Of these 392 Caucasian subjects those from whom DNA was available were included in the final case sample $(n=307)$. The control samples came from two sources. Four hundred seventy control DNA samples came from the National Institute of Mental Health (NIMH) Human Genetic Initiative (http://www. nimhgenetics.org) and were recruited by a commercial marketing firm under subcontract to NIMH to represent the general population of the United States. An additional 72 control subjects were included to increase sample size and improve power. These control subjects were recruited and screened in parallel with the PD cases (43). Additional details of the recruitment and ascertainment of these two control groups are in Methods and Materials in Supplement 1. Previous comparisons of the two control subjects sets showed that they were similar on measures of neuroticism and extraversion (43). The genotype distribution for rs3813034 in the two control samples was similar $\left(\chi^{2}=.2\right.$, $p=.9)$. All PD cases and control subjects were Caucasian by subject report, and empiric testing of ancestry informative markers demonstrated balanced population substructure in cases and control subjects (32).

\section{Genotyping}

Tissue samples and human subjects were genotyped at rs3813034 with a TaqMan 5' exonuclease assay (Applied Biosystems [ABI], Foster City, California). All samples whose initial genotype was not clear were re-genotyped in duplicate, and all such samples provided clear concordant genotypes on rerun. The 5HTTLPR, including characterization of an SNP within the polymorphic region (rs25531), was genotyped in tissue samples with a reported protocol (44). We calculated LD between the 5HTTLPR and rs3813034 in the Caucasian population with genotype data from the lymphoblast sample analyzed in Haploview (http://www.broadinstitute.org/mpg/haploview) (45).

\section{Quantitative Real-Time Polymerase Chain Reaction Analysis of SERT Polyadenylation Forms}

We developed two $5^{\prime}$ exonuclease assays to quantify total SERT mRNA and the portion of SERT messages that contained sequence $3^{\prime}$ to the proximal polyadenylation site (Figure 1). Details of assay design, optimization, and real-time polymerase chain raction (PCR) methodology are provided in Supplement 1. Glyceraldehyde-3-phosphate dehydrogenase (GAPDH) mRNA levels in lymphoblast cultures were measured with a commercially available $5^{\prime}$ exonuclease activity assay (ABI). 


\section{Transfection Studies of Polymorphic SERT 3' UTRs}

We cloned the 3' UTR of SERT into a plasmid containing the coding sequence of green fluorescent protein (GFP) as a reporter. Site-directed mutagenesis was performed to create constructs for the two alleles $(\mathrm{T} / \mathrm{G})$ of rs3813034 on otherwise identical backgrounds to ensure any differences in polyadenylation site usage were not due to polymorphisms in LD with rs3813034. Constructs were confirmed by sequencing of the complete reporter plasmids. Human embryonic kidney cells (HEK293) were used for heterologous expression of the reporter constructs, and polyadenylation site usage was determined by real-time PCR (see Supplement 1 for details of cloning, heterologous expression, and real-time PCR assays).

\section{Quantification of SERT Polyadenylation in Mouse Brain}

Brains from male and female Swiss Webster mice were obtained from Pel Freeze (Rogers, Arkansas). Real-time PCR was performed on RNA prepared from whole brain with primers targeted to the proximal and distal polyadenylation sequence. Amplification was detected with SYBR green in the PCR reactions (details of protocol and primers presented in Supplement 1).

\section{Statistical Methods}

Molecular Analyses. Statistical analyses were performed with bootstrapping with replacement of the actual data $(10,000$ replicates with Resampling Stats Excel add-in v3.2 and SAS/STAT v9.1; SAS, Cary, North Carolina). Confidence intervals (CIs) were calculated from the sampling distribution of the relevant test statistic and compared with the test statistic from the actual data to determine the $p$ values. Results outside of the $95 \%$ CI threshold were considered significant.

For tests between two groups, we performed a bootstrap analysis comparing the difference in medians across groups as the test statistic. For omnibus tests of a genotype effect, we performed a bootstrap equivalent of analysis of variance, with the $F$ statistic. For results that rejected the null hypothesis, we performed additional post hoc comparisons between two groups, with a bootstrapped multiple comparisons correction to obtain adjusted $p$ values (proc multtest function in SAS/STAT).

To test for correlation between the SERT distal polyadenylation fraction (DPF) and total SERT (mRNA), we calculated the Pearson Correlation Coefficient and performed a two-sided $t$ test (46).

\section{PD Association}

The association between PD status and genotype was analyzed in the form of a $2 \times 3$ contingency table. A $\chi^{2}$ statistic with $2 d f$ was used to test for association. If the $p$ value associated with this statistic was found to be $<.025$, we concluded that there was a significant association and computed odds ratios (ORs) for each of the genotypes $(\mathrm{G} / \mathrm{T}),(\mathrm{G} / \mathrm{G})$ compared with the genotype $(\mathrm{T} / \mathrm{T})$. We also performed a Cochran-Armitage trend test (47) to determine whether there was an increasing trend in proportion of panic patients with increasing numbers of the risk-allele.

Association between alleles and phenotype was analyzed in the form of a $2 \times 2$ contingency table with a $\chi^{2}$ statistic with $1 d f$ used to test for association. Variation of this association with gender (i.e., gender interaction) was tested with a Breslow-Day test for homogeneity of the OR (48).

Post hoc power in the association sample was calculated on the basis of the prevalence of rs3813034 alleles in the control sample and the genotype relative risks determined through the primary association analysis (49).

\section{Results}

Association of SERT Polyadenylation Forms with rs3813034

We hypothesized that, by altering one of the six nucleotides that comprise the distal polyadenylation signal (Figure 1), rs3813034 would alter the usage of that signal relative to the more $5^{\prime}$ or proximal polyadenylation signal. To test this hypothesis we developed two quantitative real-time PCR (qPCR) assays. The first assay targets coding sequence of the SERT message and quantifies total SERT mRNA in the tissue samples. The second assay targets the junction of the proximal and distal polyadenylation forms of SERT. This assay amplifies only SERT messages that contain the 123-bp distal polyadenylation sequence. With the two assays together we determined the relative portion of total SERT mRNA molecules that contain the distal polyadenylation sequence, which we term the SERT DPF. To ensure reliable quantitation of the polyadenylation forms of SERT, we optimized both qPCR assays to display highly efficient amplification across the entire range of experimental target abundance that was strongly correlated with input target abundance (Methods and Materials and Figure S2 in Supplement 1).

We applied the optimized qPCR assays to population samples of human brain tissue and lymphoblast cultures. We found that the SERT DPF in brain was significantly higher than in lymphoblasts (Figure S3 in Supplement 1). We then stratified the SERT DPF data from brain and lymphoblast samples by genotype at rs3813034 to test for association. All genotypes in brain and lymphoblast samples were in Hardy-Weinberg equilibrium. We found that in both brain tissue and lymphoblast cultures there was a highly significant and dose-dependent association of the $\mathrm{T}$ allele of rs3813034 with higher SERT DPF (Figure 2). We did not see any effect of drug exposures on the SERT DPF in brain, and drug exposures were similar across genotype groups. We also genotyped the 5HTTLPR in the brain and lymphoblast samples to test for association with the SERT DPF and found no association of the 5HTTLPR with the SERT DPF or interaction with the rs3813034 association.

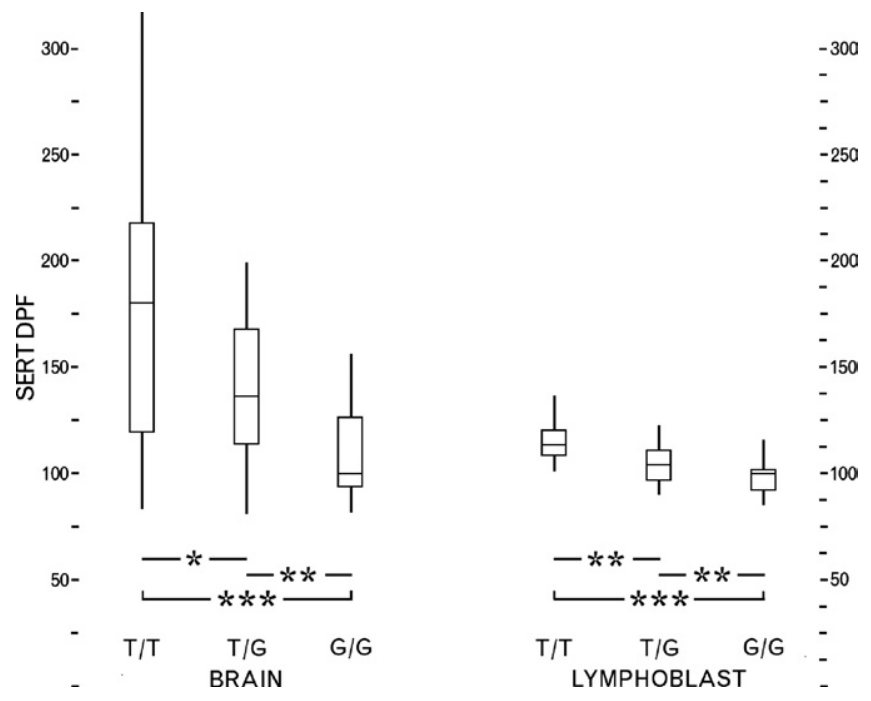

Figure 2. Distal polyadenylation fraction (DPF) stratified by rs3813034 genotype.Values are normalized to the median value for the G/G genotype separately for brain and lymphoblasts. The center line of each plot represents the median value, boxes represent the $25 \%$ and $75 \%$ percentiles, and whiskers extend to the $10 \%$ and $90 \%$ percentiles. ${ }^{*} p<.05$, ${ }^{* *} p<.01,{ }^{* * *} p<$ .001. SERT, serotonin transporter. 
To test the effects of rs3813034 on SERT polyadenylation site usage in a more controlled manner and ensure that the genotype effects we identified were not related to polymorphisms in LD with rs3813034, we cloned the 3' UTR of SERT into a GFP coding reporter plasmid. We performed site-directed mutagenesis to create two plasmids representing each allele of rs3813034 that were otherwise identical. We introduced the SERT 3 ' UTR reporter constructs into HEK293 cells and quantified the relative usage of the two polyadenylation sites. We found that the T allele of rs3813034 caused significantly higher usage of the distal polyadenylation site than the $G$ allele with a magnitude similar to the tissue samples (Figure 3).

\section{Association of SERT Polyadenylation Forms with Gender}

We stratified the qPCR data from the tissue studies by gender across all rs3813034 genotypes that displayed statistically similar distributions in male and female samples (brain: $\chi^{2}=1.34, p=$ .51; LB: $\chi^{2}=1.47, p=.48$ ). We found that the SERT DPF in brain tissue from men was significantly higher than tissue from women (Figure 4). In contrast, the SERT DPF was not associated with gender in lymphoblasts. To ensure that the gender association in brain was not an artifact due to unrelated variables that differed between male and female brain tissue, we used rapid amplification of complementary DNA ends (RACE) to determine that the two polyadenylation forms of SERT are conserved in mouse (Methods and Materials and Figure S4 in Supplement 1). We then analyzed RNA from male and female Swiss Webster mice and found that they display a gender difference that is similar to humans with a higher SERT DPF in male mouse brain than female (Figure 5).

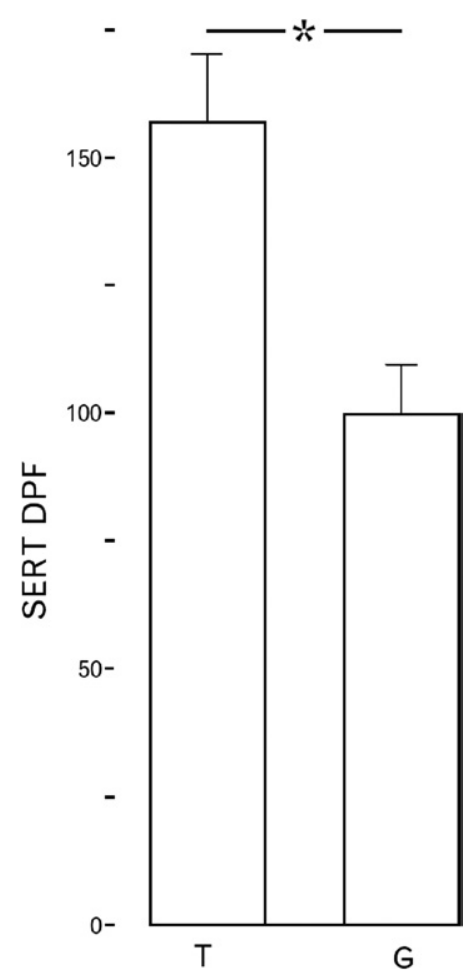

Figure 3. Confirmation of allelic effects of rs3813034 in reporter constructs heterologously expressed in HEK293 cells. Serotonin transporter (SERT) distal polyadenylation fraction (DPF) is plotted with values normalized to the median value for the $\mathrm{G}$ allele-containing construct. Error bars represent the standard error of the mean. ${ }^{*} p<.05$.

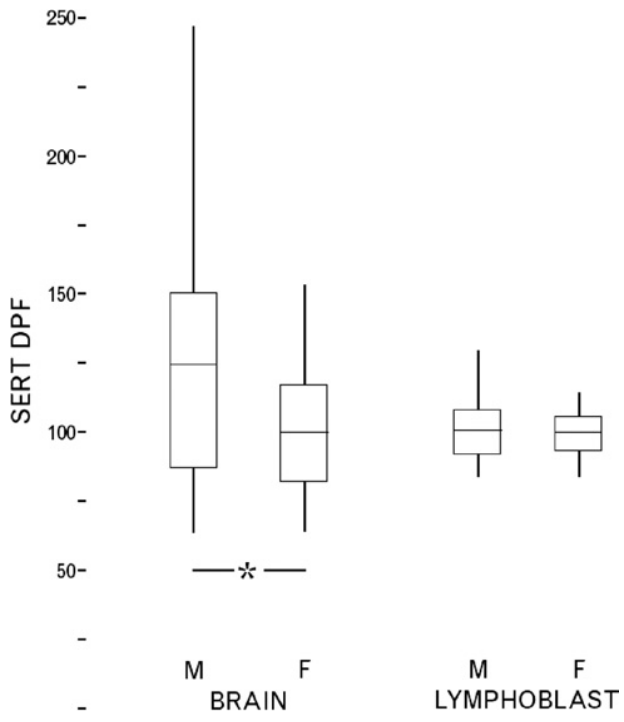

Figure 4. Distal polyadenylation fraction (DPF) stratified by gender. Values are normalized to the median value for women separately for brain and lymphoblasts. The center line of each plot represents the median value, boxes represent the $25 \%$ and $75 \%$ percentiles, and whiskers extend to the $10 \%$ and $90 \%$ percentiles. ${ }^{*} p<.05$. SERT, serotonin transporter.

To gain insight into functional effects of the distal polyadenylation sequence, we compared the SERT DPF in the lymphoblast cultures with total expression of SERT, with GAPDH as an endogenous control gene (Figure 6), and found a significant correlation $[r=.35 ; t(58)=2.85 ; p=.006]$. We did not see a significant association of rs3813034 or any of the other markers that have been associated with PD, with total SERT mRNA.

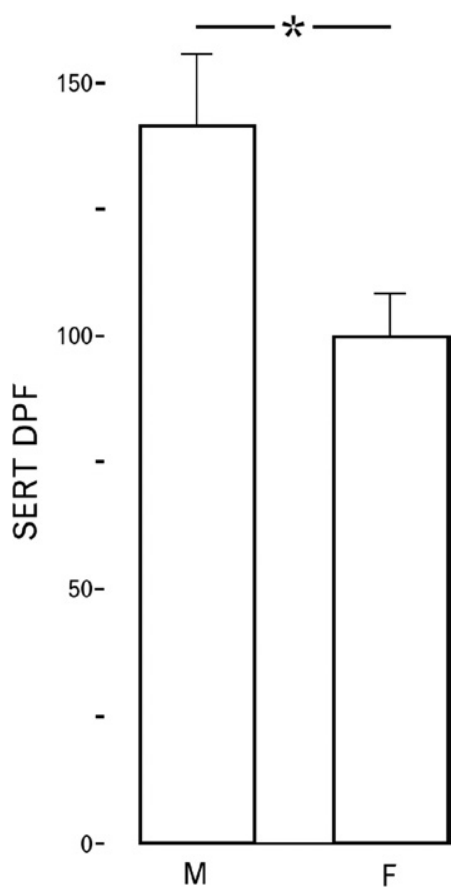

Figure 5. Confirmation of the gender effect on serotonin transporter (SERT) polyadenylation in mouse brain. The SERT distal polyadenylation fraction (DPF) in male and female brain from Swiss Webster mice (error bars represent the SEM). The SERT DPF in men are normalized to the mean value for women. ${ }^{*} p<.05$. 


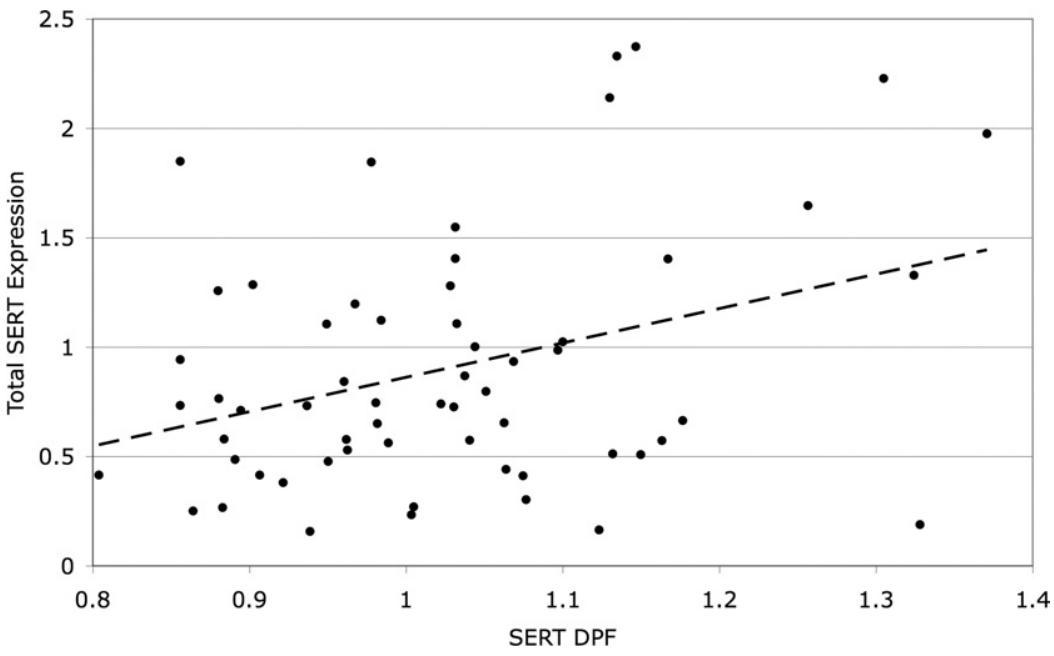

Figure 6. Correlation between the serotonin transporter (SERT) distal polyadenylation fraction (DPF) and total expression of SERT. Total expression of SERT is normalized to expression of glyceraldehyde-3-phosphate dehydrogenase as an endogenous control gene. Dashed line is the least squares fit for the data $(r=.35, p=.006)$.

\section{Association Analysis of rs3813034 with PD}

We used the information from our molecular characterization of rs3813034 to develop an a priori prediction about how it would be associated with PD. Specifically, we predicted that the $G$ allele of rs3813034 would be associated with increased risk for PD, because the G allele is associated with a lower SERT DPF, which is a similar molecular phenotype to that displayed by female brain. Panic disorder is a female-predominant disorder displaying a roughly twofold higher prevalence in women than men; so we hypothesized that, if SERT polyadenylation is meaningfully related to risk for PD, the G allele would be the risk allele. Results of the association analysis are presented in Table 1.

Table 1. Association of rs 3813034 and PD

\begin{tabular}{|c|c|c|c|c|}
\hline Genotype & GG & GT & $\pi$ & \\
\hline \multicolumn{5}{|l|}{ All } \\
\hline PD cases $n=307(\%)$ & $80(26)$ & $155(50)$ & $72(24)$ & $\chi^{2}=10.03$ \\
\hline $\begin{array}{c}\text { Control subjects } \\
n=544(\%)\end{array}$ & $112(21)$ & $250(46)$ & $182(33)$ & $p=.007$ \\
\hline \multicolumn{5}{|l|}{ Women Only } \\
\hline PD cases $n=245(\%)$ & $64(26)$ & $124(51)$ & $57(23)$ & $\chi^{2}=8.93$ \\
\hline $\begin{array}{l}\text { Control subjects } \\
n=270(\%)\end{array}$ & $51(19)$ & $126(47)$ & $93(34)$ & $p=.012$ \\
\hline \multicolumn{5}{|l|}{ Men Only } \\
\hline PD cases $n=62(\%)$ & $16(26)$ & $31(50)$ & $15(24)$ & $\chi^{2}=1.65$ \\
\hline $\begin{array}{c}\text { Control subjects } \\
n=274(\%)\end{array}$ & $61(22)$ & $124(45)$ & $89(33)$ & $p=.439$ \\
\hline Allele & G & $\mathrm{T}$ & Odds Ratio & \\
\hline \multicolumn{5}{|l|}{ All } \\
\hline PD cases $n=614(\%)$ & $315(51)$ & $299(49)$ & 1.36 & $\chi^{2}=9.45$ \\
\hline $\begin{array}{c}\text { Control subjects } \\
\mathrm{n}=1088(\%)\end{array}$ & $474(44)$ & $614(56)$ & $(1.12-1.66)$ & $p=.002$ \\
\hline \multicolumn{5}{|l|}{ Women Only } \\
\hline PD cases $n=490(\%)$ & $252(51)$ & $238(49)$ & 1.45 & $\chi^{2}=8.75$ \\
\hline $\begin{array}{l}\text { Control subjects } \\
n=540(\%)\end{array}$ & $228(42)$ & $312(58)$ & $(1.13-1.85)$ & $\mathrm{p}=.003$ \\
\hline \multicolumn{5}{|l|}{ Men Only } \\
\hline PD cases $n=124(\%)$ & $63(51)$ & 61 (49) & 1.27 & $\chi^{2}=1.42$ \\
\hline $\begin{array}{l}\text { Control subjects } \\
n=548(\%)\end{array}$ & $246(45)$ & $302(55)$ & $(0.86-1.87)$ & $p=.233$ \\
\hline
\end{tabular}

$\mathrm{PD}$, panic disorder.
Genotype analysis of rs3813034 in PD cases and control subjects identified a statistically significant association between phenotype and genotype: $\left(\chi^{2}=10.03, p=.0068\right)$. Odds ratios comparing proportions of panic patients with genotypes $(G, T)$ and $(G, G)$ with proportions of patients with genotype (T, T) were as follows: 1.56 (95\% CI: 1.12-2.20), 1.81 (95\% CI: $1.22-$ 2.68). The Cochran-Armitage test for trend was highly significant ( $z=3.0095, p=.0025$ ), implying that there is an increasing linear trend in the proportion of panic patients with increasing numbers of the $G$-allele.

When analyzed separately by gender it was found that there was a significant association between phenotype and genotype in women: $\left(\chi^{2}=8.93, p=.0115\right)$ and ORs comparing genotypes $(\mathrm{G} / \mathrm{T})$ and $(\mathrm{G} / \mathrm{G})$ with genotype $(\mathrm{T} / \mathrm{T})$ were found to be 1.61 (95\% CI: 1.06-2.43) and 2.05 (95\% CI: 1.25-3.36), respectively. In addition, for women the Cochran-Armitage test for trend was significant $(z=2.9222, p=.0115)$, implying that there is an increasing linear trend in the proportion of female panic patients with increasing numbers of the $G$ allele. There was no significant association found between phenotype and genotype in men $\left(\chi^{2}=1.6459, p=.4391\right)$. Furthermore, a test for trend with the Cochran-Armitage test was not significant $(z=-1.1532, p=$ .2488). Post hoc analysis on the basis of the empiric genotype relative risks determined that the full sample (women and men) has $>90 \%$ power to identify a nominal association at an $\alpha$ value of .05 (49).

Allele-based analysis of rs3813034 in PD cases and control subjects identified a statistically significant association between phenotype and allele in the total sample: $\chi^{2}=9.45, p=.0021$. The OR corresponding to this association was 1.3647 , and the associated 95\% CI was found to be 1.1190-1.6643. When the sample was analyzed separately by gender it was found that the association between phenotype and allele for women was statistically significant with $\chi^{2}=8.7498, p=.0031$. The OR corresponding to this association was found to be 1.4489 (95\% CI: 1.1329-1.8531). For men, this association was not found to be statistically significant: $\chi^{2}=1.4248, p=.2326$. The corresponding OR was found to be 1.2679 ( $95 \%$ CI: .8582-1.8731). When a formal test of interaction to determine whether these ORs varied by gender was conducted with the Breslow-Day test for homogeneity, a statistically significant interaction was not found $\left(\chi^{2}=.3216, p=\right.$ .5707). 
The 5HTTLPR and rs3813034 are in low LD $\left(D^{\prime}=.27, r^{2}=\right.$ .06 in the LB samples) and might therefore confer risk for PD independently. This sample has previously been tested for an association with the 5HTTLPR, which was negative, and we did not see an interaction between the 5HTTLPR and rs3813034 (32).

\section{Discussion}

In this report, we have identified a molecular phenotype for rs3813034 and demonstrated that it is associated with PD. We have determined that the $\mathrm{T}$ allele of rs3813034 leads to more efficient usage of the distal polyadenylation signal in which it occurs than the $G$ allele. This is consistent with in vitro studies of polyadenylation that have shown that a $T$ in the position of rs3813034 within the canonical polyadenylation signal (AAUAAA) leads to more efficient polyadenylation than a $G$ (50). Our observation that rs3813034 affects the balance of SERT polyadenylation forms in brain, lymphoblast cultures, and heterologous expression experiments provides confidence that the molecular phenotype of rs3813034 is both reliable and potentially relevant to behavioral variability.

In contrast to the polymorphic effects of rs3813034, the gender difference in SERT polyadenylation was only present in brain and not lymphoblasts. This is likely because lymphoblast cultures are grown under identical conditions regardless of the gender of the individual from whom the cells were derived and that gender-specific hormones influence the SERT DPF. This possibility will require further studies, which we are conducting. To provide additional confidence that this gender effect was not spurious, we tested brains from Swiss Webster mice and found a similar gender effect on the SERT DPF.

Our biological characterization of SERT polyadenylation allowed us to refine the reported association between markers in SERT and PD in this sample (32). We made a directional prediction that the $G$ allele of rs3813034 would be the risk allele for PD, because the G allele mimics the effects of female gender, which is itself a risk factor for PD. We confirmed this prediction, demonstrating that the $G$ allele of rs3813034 confers increased risk for PD. The gender difference in the SERT DPF in brain also led us to predict that the risk effect of the G allele of rs3813034 would be greater in women than men, because women display a lower SERT DPF in brain independent of genotype at rs381034, and therefore risk alleles of rs3813034 would represent second and/or third hits conferring greater risk on women than men. This prediction is somewhat supported by our data, because we did identify a greater apparent risk effect of the $G$ allele in women than men (Table 1), but the estimation of the risk effect in men is tentative because the overall association in men was not significant. We note that the association we report is not an independent replication of the previously reported association between SERT and PD with the sample we tested (32). Rather we propose that rs3813034 is the risk factor that explains the reported association to markers with which it is in high LD.

Although we do not know all the different biological attributes of SERT mRNA molecules that are proximally versus distally polyadenylated, we did find that the SERT DPF is correlated with total SERT expression, suggesting that the distal sequence stabilizes SERT mRNA either directly through effects on the secondary structure of the message or through interactions with RNA binding proteins or microRNAs. Bioinformatic analysis of the distal sequence identifies putative targets for RNA binding proteins and/or microRNAs that can affect message stability, translational activity, and subcellular localization, but these algo- rithms are not adequately reliable to make confident predictions. Nonetheless, the high degree of sequence similarity of the proximal and distal polyadenylation forms of SERT between human and mouse (Figure S4 in Supplement 1) is de facto evidence that both forms have important evolutionarily conserved functions (51). Mechanistic studies to identify the biological functions of the distal sequence are under way and might help further guide association studies of the role of rs3813034 in psychopathology.

Dysregulation of serotonin neurotransmission has been implicated in the pathophysiology of PD (7). The SSRIs are effective treatments for PD and selectively target SERT (52-54). Serotonin turnover, which is in part related to SERT activity, is increased in the brain of PD subjects and normalizes with SSRI treatment (15). SERT binding in platelets from PD subjects is reduced and also normalizes with SSRI or tricyclic antidepressant treatment (55). Similarly, SERT binding in the brains of subjects with current PD is decreased in raphe, temporal cortex, and thalamus and normalizes in PD subjects in remission in the raphe and temporal cortex (56). These data might be explained at least in part by our finding that a low SERT DPF seems to be a risk factor for PD and is also correlated with lower expression of SERT mRNA. Further studies will be required to test this possibility directly, because mRNA levels do not necessarily predict protein levels. There have been reports that a polymorphism adjacent to rs3813034 (rs1042173) is functional, and this polymorphism is one of those previously reported to be associated with PD in this sample $(32,57,58)$. rs3813034 and rs1042173 are in absolute LD in Caucasians, and therefore any independent PD risk effects of these two polymorphisms cannot be addressed in this study. We note, however, that the reported functional studies of rs1042173 used only in vitro analyses to identify a molecular phenotype and found conflicting effects of the alleles of rs10422173 on SERT expression $(57,58)$. The problem of identifying the specific risk factor among highly correlated polymorphisms is an unavoidable challenge in complex trait genetics. We believe that intensive characterization of the molecular and cellular effects of candidate risk factors with the most biologically representative approaches is one way to address this problem.

A limitation of the PD association study is that the control subjects came from two sources and were recruited and ascertained with different methods. The cases were also recruited and ascertained differently than the bulk of the control subjects. We believe that the impact of this possible source of heterogeneity is minimal, because the genotype distribution of rs3813034 in the two control samples is similar and the case/control sample was empirically determined to have balanced population structure. The present association of $\mathrm{rs} 3813034$ with PD will require replication before it can be considered a risk factor for PD and/or other serotonin transporter-related behavioral disorders, but several aspects of this study suggest the association is valid: 1) the molecular characterization of rs3813034 demonstrates that it is a functional polymorphism; 2) the directional prediction we made on the basis of the molecular characterization of rs3813034 was confirmed by association testing; 3) this sample has a balanced population structure between cases and control subjects that was determined empirically, minimizing the risk that the observed association is due to population stratification; 4) the association displays a dose effect of risk alleles at rs3813034; and 5) the case-control sample has been rigorously ascertained and is well-powered to identify an association of the observed effect size to a common polymorphism such as rs3813034. 
The National Institute of Mental Health control sample was collected by "Molecular Genetics of Schizophrenia II" collaboration and included the following investigators: Evanston Northwestern Healthcare/Northwestern University, Evanston, Illinois, MH059571-Pablo V. Gejman, M.D. (Collaboration Coordinator; principle investigator [PI]), Alan R Sanders, M.D.; Emory University School of Medicine, Atlanta, Georgia, MH59587Farooq Amin, M.D. (PI); Louisiana State University Health Sciences Center, New Orleans, Louisiana, MH067257-Nancy Buccola APRN, BC, MSN (PI); University of California-Irvine, Irvine, California, MH60870-William Byerley, M.D. (PI); Washington University, St Louis, Missouri, U01, MH060879-C Robert Cloninger, MD (PI); University of Iowa, Iowa City, Iowa, MH59566-Raymond Crowe, M.D. (PI), Donald Black, M.D.; University of Colorado, Denver, Colorado, MH059565-Robert Freedman, M.D. (PI); University of Pennsylvania, Philadelphia, Pennsylvania, MH061675-Douglas Levinson, M.D. (PI); University of Queensland, QLD, Australia, MH059588-Bryan Mowry, M.D. (PI); Mt Sinai School of Medicine, New York, New York, MH59586-Jeremy Silverman, Ph.D. (PI).

This work was supported in part by: T32 MH65213 (RS); PO1 MH60970 (R. Hen PI), Sackler Institute for Developmental Psychobiology at Columbia University (MMW); MH48858 (SEH); and the Hartwell Foundation, the Drukier Foundation, MH79513 (CEG).

In the past 2 years, the authors have received research funding from the National Institute of Mental Health (CEG, $M M W)$, National Institute for Drug Abuse (CEG, MJB), and the National Alliance for Research on Schizophrenia and Depression (CEG). MMW receives royalties from the Oxford University Press, Perseus Press, the American Psychiatric Association, and MultiHealth Systems. All other authors report no biomedical financial interests or potential conflicts of interest. online.

Supplementary material cited in this article is available

1. Weissman MM, Bland RC, Canino GJ, Faravelli C, Greenwald S, Hwu HG, et al. (1997): The cross-national epidemiology of panic disorder. Arch Gen Psychiatry 54:305-309.

2. Crowe RR, Noyes R, Pauls DL, Slymen D (1983): A family study of panic disorder. Arch Gen Psychiatry 40:1065-1069.

3. Noyes R Jr, Crowe RR, Harris EL, Hamra BJ, McChesney CM, Chaudhry DR (1986): Relationship between panic disorder and agoraphobia. A family study. Arch Gen Psychiatry 43:227-232.

4. Hopper JL, Judd FK, Derrick PL, Macaskill GT, Burrows GD (1990): A family study of panic disorder: Reanalysis using a regressive logistic model that incorporates a sibship environment. Genet Epidemiol 7:151-161.

5. Hettema JM, Neale MC, Kendler KS (2001): A review and meta-analysis of the genetic epidemiology of anxiety disorders. Am J Psychiatry 158: $1568-1578$.

6. Smoller JW, Gardner-Schuster E, Covino J (2008): The genetic basis of panic and phobic anxiety disorders. Am J Med Genet C Semin Med Genet 148C:118-126.

7. Maron E, Shlik J (2006): Serotonin function in panic disorder: Important, but why? Neuropsychopharmacology 31:1-11.

8. Pollack MH, Otto MW, Worthington JJ, Manfro GG, Wolkow R (1998): Sertraline in the treatment of panic disorder: A flexible-dose multicenter trial. Arch Gen Psychiatry 55:1010-1016.

9. Bakker A, van Balkom AJ, van Dyck R (2000): Selective serotonin reuptake inhibitors in the treatment of panic disorder and agoraphobia. Int Clin Psychopharmacol 15(suppl 2):S25-S30.

10. Bell CJ, Nutt DJ (1998): Serotonin and panic. Br J Psychiatry 172:465-471.

11. Holmes A, Yang RJ, Lesch KP, Crawley JN, Murphy DL (2003): Mice lacking the serotonin transporter exhibit 5-HT(1A) receptor-mediated abnormalities in tests for anxiety-like behavior. Neuropsychopharmacology 28:2077-2088.
12. Holmes A, Murphy DL, Crawley JN (2003): Abnormal behavioral phenotypes of serotonin transporter knockout mice: Parallels with human anxiety and depression. Biol Psychiatry 54:953-959.

13. Holmes A, Yang RJ, Murphy DL, Crawley JN (2002): Evaluation of antidepressant-related behavioral responses in mice lacking the serotonin transporter. Neuropsychopharmacology 27:914-923.

14. Talbot PS (2004): The molecular neuroimaging of anxiety disorders. Curr Psychiatry Rep 6:274-279.

15. Esler M, Lambert E, Alvarenga M, Socratous F, Richards J, Barton D, et al. (2007): Increased brain serotonin turnover in panic disorder patients in the absence of a panic attack: Reduction by a selective serotonin reuptake inhibitor. Stress 10:295-304.

16. Lesch KP, Bengel D, Heils A, Sabol SZ, Greenberg BD, Petri S, et al. (1996): Association of anxiety-related traits with a polymorphism in the serotonin transporter gene regulatory region. Science 274:1527-1531.

17. Heils A, Teufel A, Petri S, Stober G, Riederer P, Bengel D, et al. (1996): Allelic variation of human serotonin transporter gene expression. J Neurochem 66:2621-2624.

18. Glatt CE, Freimer NB (2002): Association analysis of candidate genes for neuropsychiatric disease: The perpetual campaign. Trends Genet 18: 307-312.

19. Hariri AR, Mattay VS, Tessitore A, Kolachana B, Fera F, Goldman D, et al. (2002): Serotonin transporter genetic variation and the response of the human amygdala. Science 297:400-403.

20. Caspi A, Sugden K, Moffitt TE, Taylor A, Craig IW, Harrington H, et al. (2003): Influence of life stress on depression: Moderation by a polymorphism in the 5-HTT gene. Science 301:386-389.

21. Olesen OF, Bennike B, Hansen ES, Koefoed P, Woldbye DP, Bolwig TG, et al. (2005): The short/long polymorphism in the serotonin transporter gene promoter is not associated with panic disorder in a Scandinavian sample. Psychiatr Genet 15:159.

22. Samochowiec J, Hajduk A, Samochowiec A, Horodnicki J, Stepien G, Grzywacz A, et al. (2004): Association studies of MAO-A, COMT, and 5-HTT genes polymorphisms in patients with anxiety disorders of the phobic spectrum. Psychiatry Res 128:21-26.

23. Hamilton SP, Heiman GA, Haghighi F, Mick S, Klein DF, Hodge SE, et al. (1999): Lack of genetic linkage or association between a functional serotonin transporter polymorphism and panic disorder. Psychiatr Genet 9:1-6.

24. Deckert J, Catalano M, Heils A, Di Bella D, Friess F, Politi E, et al. (1997): Functional promoter polymorphism of the human serotonin transporter: Lack of association with panic disorder. Psychiatr Genet 7:45-47.

25. Ishiguro H, Arinami T, Yamada K, Otsuka Y, Toru M, Shibuya H (1997): An association study between a transcriptional polymorphism in the serotonin transporter gene and panic disorder in a Japanese population. Psychiatry Clin Neurosci 51:333-335.

26. Maron E, Lang A, Tasa G, Liivlaid L, Toru I, Must A, et al. (2005): Associations between serotonin-related gene polymorphisms and panic disorder. Int J Neuropsychopharmacol 8:261-266.

27. Ohara K, Nagai M, Suzuki Y, Ochiai M, Ohara K (1998): Association between anxiety disorders and a functional polymorphism in the serotonin transporter gene. Psychiatry Res 81:277-279.

28. Martinez-Barrondo S, Saiz PA, Morales B, Garcia-Portilla MP, Coto E, Alvarez V, et al. (2005): Serotonin gene polymorphisms in patients with panic disorder. Actas Esp Psiquiatr 33:210-215.

29. Kim W, Choi YH, Yoon KS, Cho DY, Pae CU, Woo JM (2006): Tryptophan hydroxylase and serotonin transporter gene polymorphism does not affect the diagnosis, clinical features and treatment outcome of panic disorder in the Korean population. Prog Neuropsychopharmacol Biol Psychiatry 30:1413-1418.

30. Matsushita S, Muramatsu T, Kimura M, Shirakawa O, Mita T, Nakai T, et al. (1997): Serotonin transporter gene regulatory region polymorphism and panic disorder. Mol Psychiatry 2:390-392.

31. Blaya C, Salum GA, Lima MS, Leistner-Segal S, Manfro GG (2007): Lack of association between the serotonin transporter promoter polymorphism (5-HTTLPR) and panic disorder: A systematic review and metaanalysis. Behav Brain Funct 3:41.

32. Strug L, Suresh R, Fyer AJ, Talati A, Adams PB, Li W, et al. (2008): Panic disorder is associated with the serotonin transporter gene (SLC6A4) but not the promoter region (5-HTTLPR) [published online ahead of print July 29]. Mol Psychiatry.

33. Battersby S, Ogilvie AD, Blackwood DH, Shen S, Muqit MM, Muir WJ, et al. (1999): Presence of multiple functional polyadenylation signals and a 
single nucleotide polymorphism in the $3^{\prime}$ untranslated region of the human serotonin transporter gene. J Neurochem 72:1384-1388.

34. Heils A, Teufel A, Petri S, Seemann M, Bengel D, Balling U, et al. (1995): Functional promoter and polyadenylation site mapping of the human serotonin (5-HT) transporter gene. J Neural Transm Gen Sect 102:247254.

35. Talati A, Ponniah K, Strug LJ, Hodge SE, Fyer AJ, Weissman MM (2008): Panic disorder, social anxiety disorder, and a possible medical syndrome previously linked to chromosome 13. Biol Psychiatry 63:594601.

36. Albertson DN, Pruetz B, Schmidt CJ, Kuhn DM, Kapatos G, Bannon MJ (2004): Gene expression profile of the nucleus accumbens of human cocaine abusers: Evidence for dysregulation of myelin. J Neurochem 88:1211-1219.

37. Albertson DN, Schmidt CJ, Kapatos G, Bannon MJ (2006): Distinctive profiles of gene expression in the human nucleus accumbens associated with cocaine and heroin abuse. Neuropsychopharmacology 31: $2304-2312$.

38. Kingsbury AE, Foster OJ, Nisbet AP, Cairns N, Bray L, Eve DJ, et al. (1995): Tissue $\mathrm{pH}$ as an indicator of mRNA preservation in human post-mortem brain. Brain Res Mol Brain Res 28:311-318.

39. Fyer AJ, Endicott J, Mannuzza S, Klein DF (1985): Schedule for Affective Disorders and Schizophrenia-Lifetime Version Modified for the Study of Anxiety Disorders (SADS-LA). New York, New York: State Psychiatric Institute.

40. Mannuzza S, Fyer AJ, Klein DF, Endicott J (1986): Schedule for affective disorders and schizophrenia-Lifetime version modified for the study of anxiety disorders (SADS-LA): Rationale and conceptual development. J Psychiatr Res 20:317-325.

41. Weissman MM, Wickramaratne $P$, Adams $P$, Wolk S, Verdeli $H$, Olfson M (2000): Brief screening for family psychiatric history: The family history screen. Arch Gen Psychiatry 57:675-682.

42. Weissman MM (1993): Family genetic studies of panic disorder. J Psychiatr Res 27(suppl 1):69-78.

43. Talati A, Fyer AJ, Weissman MM (2008): A comparison between screened NIMH and clinically interviewed control samples on neuroticism and extraversion. Mol Psychiatry 13:122-130.

44. Wendland JR, Martin BJ, Kruse MR, Lesch KP, Murphy DL (2006): Simultaneous genotyping of four functional loci of human SLC6A4, with a reappraisal of 5-HTTLPR and rs25531. Mol Psychiatry 11:224-226.
45. Barrett JC, Fry B, Maller J, Daly MJ (2005): Haploview: Analysis and visualization of $L D$ and haplotype maps. Bioinformatics 21:263-265.

46. Wessa P (2009): Free Statistics Software, Office for Research Development and Education, version 1.1.23-r4. Available at: http://www.wessa. net/. Accessed November 20, 2009.

47. Agresti A (1990): Categorical Data Analysis. New York: John Wiley \& Sons.

48. Breslow NE, Day NE (1980): Statistical methods in cancer research. Vol I-The analysis of case-control studies. IARC Sci Publ 5-338.

49. Purcell S, Cherny SS, Sham PC (2003): Genetic power calculator: Design of linkage and association genetic mapping studies of complex traits. Bioinformatics 19:149-150.

50. Sheets MD, Ogg SC, Wickens MP (1990): Point mutations in AAUAAA and the poly(A) addition site: Effects on the accuracy and efficiency of cleavage and polyadenylation in vitro. Nucleic Acids Res 18:5799-5805.

51. Xie X, Lu J, Kulbokas EJ, Golub TR, Mootha V, Lindblad-Toh K, et al. (2005): Systematic discovery of regulatory motifs in human promoters and $3^{\prime}$ UTRs by comparison of several mammals. Nature 434:338-345.

52. Boyer W (1995): Serotonin uptake inhibitors are superior to imipramine and alprazolam in alleviating panic attacks: A meta-analysis. Int Clin Psychopharmacol 10:45-49.

53. Lecrubier Y, Bakker A, Dunbar G, Judge R (1997): A comparison of paroxetine, clomipramine and placebo in the treatment of panic disorder. Collaborative Paroxetine Panic Study Investigators. Acta Psychiatr Scand 95:145-152.

54. Lecrubier Y, Judge R (1997): Long-term evaluation of paroxetine, clomipramine and placebo in panic disorder. Collaborative Paroxetine Panic Study Investigators. Acta Psychiatr Scand 95:153-160.

55. Marazziti D, Rossi A, Dell'Osso L, Palego L, Placidi GP, Giannaccini G, et al. (1999): Decreased platelet 3H-paroxetine binding in untreated panic disorder patients. Life Sci 65:2735-2741.

56. Maron E, Kuikka JT, Shlik J, Vasar V, Vanninen E, Tiihonen J (2004): Reduced brain serotonin transporter binding in patients with panic disorder. Psychiatry Res 132:173-181.

57. Seneviratne C, Huang W, Ait-Daoud N, Li MD, Johnson BA (2009): Characterization of a functional polymorphism in the 3' UTR of SLC6A4 and its association with drinking intensity. Alcohol Clin Exp Res 33:332-339.

58. Vallender EJ, Priddy CM, Hakim S, Yang H, Chen GL, Miller GM (2008): Functional variation in the $3^{\prime}$ untranslated region of the serotonin transporter in human and rhesus macaque. Genes Brain Behav 7:690-697. 